\title{
SENTIMENT ANALYSIS OF KAI ACCESS APPLICATION USING THE DEEP NEURAL NETWORK METHOD
}

\author{
Prima Dina Atika ${ }^{1}$, Herlawati², Fata Nidaul Khasanah ${ }^{3}$, Rafika Sari ${ }^{4}$ \\ Informatics, Department, Bhayangkara Jakarta Raya University, Jakarta, Indonesia ${ }^{1-4}$
}

\begin{abstract}
Fire mode of transportation is a mode of transportation that can overcome traffic jams The mode of transportation in Indonesia is managed by PT Kereta Api Indonesia (KAI). From day PT KAI always improves services to the community. To know how much people comment positively and negatively, a sentiment analysis is carried out against PT KAI. By retrieving data by scraping from the page Google Play website. The data obtained is data from the Google Play website database which has as many as 600 comments in the form of user reviews of KAI Access. From review KAI Access users are classified using a deep neural network. Expected From the results of this sentiment analysis, it will improve PT KAI's services
\end{abstract}

Keywords: Sentiment Analysis, CNN, PT KAI, Google play

\section{INTRODUCTION}

Along with the development of the number of vehicles that are not followed by developments a significant number of roads causing traffic jams. Public transportation is one solution to overcome the problems above. The public transport system user-friendliness is needed by the community, namely trains. The train is one of the oldest modes of transportation in the world, has various comparative and competitive advantages, can save money land and energy, have less pollution, have good properties, and adapt with changes in technology. Previously, several studies have been conducted to analyse sentiment towards Indonesian Railways Like the Naïve Bayes Classifier (NBC) method that achieves accuracy by $84 \%$. Sentiment analysis is also applied to motorcycle taxi services online using the Support Vector Machine (SVM) algorithm with an accuracy value of 76\%. The Logistics Regression Algorithm has also been successfully used to analysis of customer sentiment in banking services with an accuracy of $99.3 \%$ [1].

One of the classification algorithms that is often used and gets a lot of attention researchers are nave bayes classifier, simplicity in nave bayes algorithm that makes the algorithm has the attractiveness to be implemented in a variety of applications application, but the weakness faced by this algorithm is the length of time and the level of prediction accuracy used to make predictions[2]. The Support Vector Machine algorithm has advantages in showing performance which is very good for time series prediction [3]SVM method not produce accurate results when many features are irrelevant, not all features needed in the process [3] Logistic Regression is a linear classification which has been shown to produce a powerful classification with probability statistics and deal with multiclass classification problems. Big problem experienced by the Logistics Regression algorithm is the class imbalance (Class Balance) on high-dimensional datasets .Therefore, this study tries to apply a deep neural network for sentiment analysis contained in the KAI Access application on the Google Playstore platform, to find out response from users to the KAI Access application. The purpose of this research is to know the analysis of some sentiment data that can be used to predict the number of negative and positive reviews related to the KAI Access application using Deep Neural Network method.

Convolutional Neural Network is a Multi Layer Perceptron (MLP) development method designed to process twodimensional data. CNN is included in the type of Deep Neural Network because of the depth of the network level and is widely implemented in image data. CNN has two methods; namely the classification using feedforward and the learning stage using backpropagation. The way CNN works is similar to MLP, but in CNN each neuron is represented in two dimensions, unlike MLP where each neuron is only one size. dimensions [4] Sentiment analysis research by entering behavioural information user from [5] entitled 'Twitter Sentiment Analysis with a Deep Neural Network: An Enhanced Approach using User Behavioral Information'. This study applies the Convolutional Neural Network algorithm from the SemEval-2016 1 and SemEval-2016 2 datasets. Classical machine learning methods such as Support Vector Machine, Naïve Bayes, K-Nearest Neighbor, J48 is used for comparison of CNN algorithm. The results obtained by the CNN algorithm show consistently good performance in both datasets so that the resulting accuracy is more consistent.

Sentiment analysis based on Word2Vec was carried out by [6] in a study entitled 'Sentiment Analysis of Indonesian Twitter Based on Word2vec Using Deep Convolutional Neural Networks'. This study uses the Word2Vec model for Indonesian as a vector representation of words. Using Word2Vec also speeds up the training process and improves the accuracy of the Deep Convolutional Neural Network algorithm. The results of the experiments that have been carried out 


\section{International Journal of Advanced Research in Computer and Communication Engineering}

Vol. 10, Issue 12, December 2021

DOI: $10.17148 /$ IJARCCE.2021.101205

have resulted in an accuracy of $76.40 \%$. The purpose of this study is to find out the analysis of some sentiment data that can be used to predict the number of negative and positive reviews related to the KAI Access application using the Deep Neural Network method.

\section{RESEARCH METHODOLOGY}

The main problem in the classification of sentiments is how to identify sentiments in a text, whether the text has positive or negative sentiments[7]. Figure 1 is the flow applied in this study. The first step is retrieval of data or crawling data from twitter, then all data is labelled into two classes, positive and negative. The next step is preprocessing using NLP techniques. The results of pre-processing in the form of tokens will be input to the classification process. The classification process uses a deep learning approach with the Convolution Neural Network (CNN) algorithm. Figure 1. Research Steps.

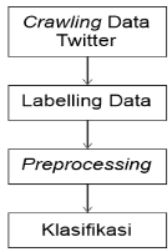

Figure 1. CNN Classification Stages

\subsection{CrawlingData}

The data is taken from the KAI web using the Python programming language, with the . A total of 1000 data were selected with search keywords including "PT KAI", "service", "application" from the KAI website.

2.2. Labelling data

Labelling the dataset is done manually with the help of experts. Data are labelled positive and negative. From the labelling results, there were 658 data with negative sentiment and 242 data with positive sentiment.

2.3 Pre-processing

The data obtained from Twitter are generally in the form of unstructured sentences such as non-alphanumeric characters, url links, irregular grammar, etc. The presence of noise and data irregularity affects the performance of the machine [14. Pre-processing NLP is a step to remove unwanted noise from the Twitter dataset [15], so that the resulting data becomes more structured.

2.3.1 Stop word Removal

stop word is a step to eliminate words that have no effect/uninformative but often appear in documents such as url links, hashtags, usernames, punctuation marks and non-alphanumeric characters (except spaces) such as! @ \# \& \& dl. Removal via python's regular expression library.

2.3.2 Tokenization

A process of cutting or parsing text into words. The result of this process is called token [20].

\section{RESULT AND DISCUSSION}

\subsection{Building a CNN Model}

Below is the python coding for building CNN by defining the embedding layer vocal size $=100$, CNN 12 model with activation is Relu and using global MAXPOOLING to produce the CNN model in Figure 2.

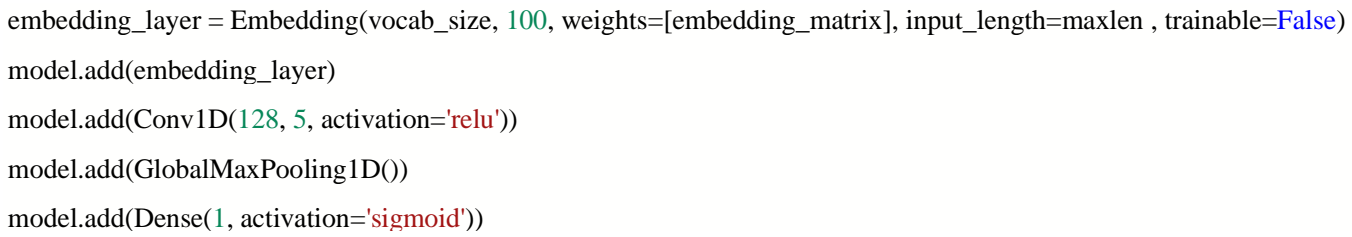


Vol. 10, Issue 12, December 2021

DOI: $10.17148 /$ IJARCCE.2021.101205

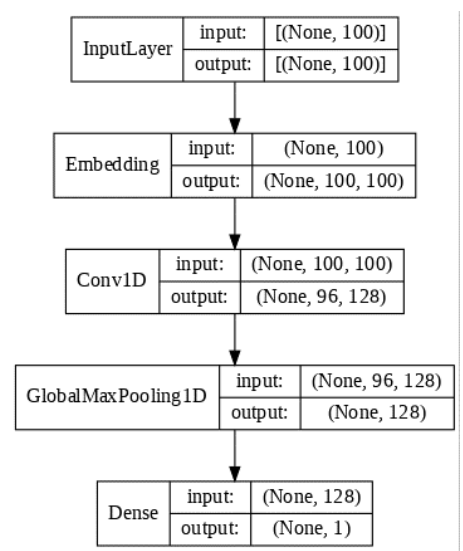

Figure 2. CNN Model

\subsection{Testing Model}

At this stage, a training model is carried out which aims to test the model on the training data with parameters for each model that has been made. Based on the parameter testing that has been tried, the model with the best parameter values is the CNN-LSTM model. Model testing produces accuracy and loss values in training and validation data. The training process uses 10 epochs and 128 batch sizes. With the parameters that have been determined, it will be seen how accurate the training data is and see the lowest loss value. The model will store the optimal epoch at the lowest loss value during the epoch process. The following are the results of model testing can be seen in Figure 3.

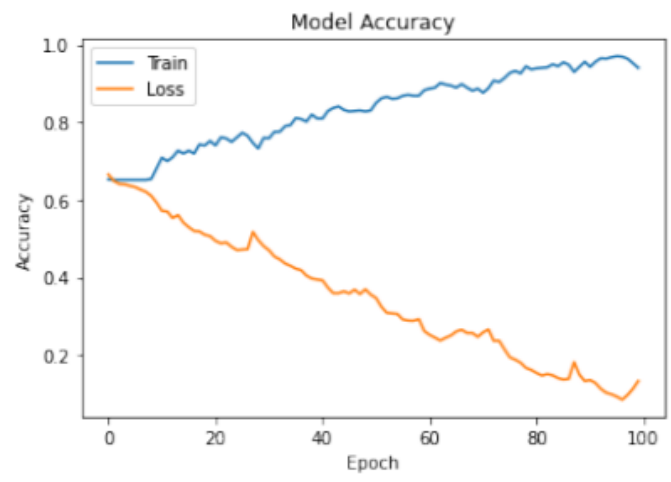

Figure 3. CNN and LSTM accuracy models

Based on Figure 3, the best test results are shown in Figure 2. The CNN-LSTM model produces the lowest accuracy value in the first epoch validation data with a value of 0.8686 and the highest loss value in the training data produces 0.762. In epoch 1 , the CNN-LSTM model produces good accuracy compared to other models. The comparison shows that the model is quite good by showing that the accuracy of the training data with validation is not much different so that it does not experience overfitting. Furthermore, the reliability of the model will be tested at the testing stage with data testing.

\subsection{Testing process}

This stage is the stage of testing the model with data testing. The data to be tested are 300 article titles with the number of labels in the negative class, 658 article titles, 242 titles in the positive class. Furthermore, after the model produces its predictions in each class, the confidence level of the model is calculated by looking at accuracy, precision and recall. The goal is to find out how reliable the model is in predicting the class. The results of testing data testing are shown by a confusion matrix as shown in the table below. Table 1 shows the results of the Confusion Matrix.

Table 1. Results of the Confusion Matrix

\begin{tabular}{|l|l|l|}
\hline \multirow{2}{*}{ Actual } & Prediction \\
\cline { 2 - 3 } & Negative & Positive \\
\hline Negative & 33 & 102 \\
\hline Positive & 45 & 120 \\
\hline
\end{tabular}


Based on the model that has been made where the test data labelled as negative is predicted to be negative at 33 and correctly predicts 102 and the actual positive one predicts 45 . Furthermore, after the model produces its predictions in each class, the confidence level of the model is calculated by looking at the accuracy, precision and recalls. The goal is to find out how much the percentage of accuracy can be trusted as a model in predicting class. Figure 4 to see accuracy, precision, recall.

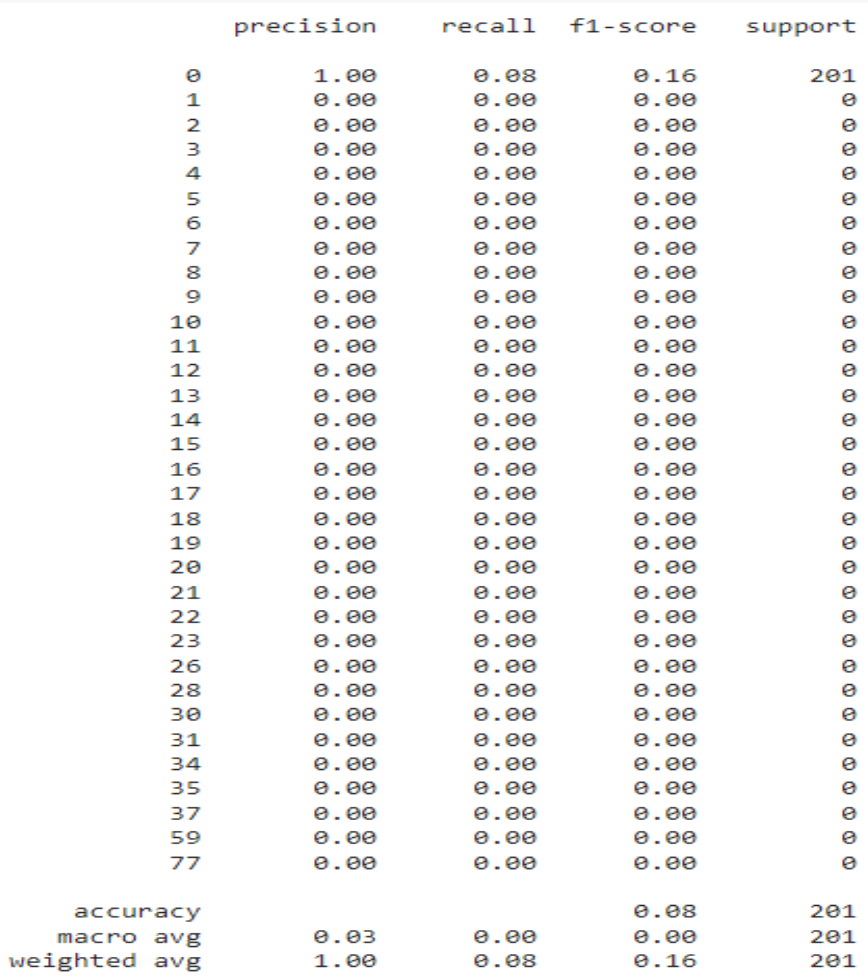

Figure 4. Accuracy, precision, Recall

\section{CONCLUSION}

After going through the stage of testing the level of performance with the parameters of accuracy, precision, recall on the CNN model in determining sentiment analysis, predictions of using the KAI application, the conclusions include:

1. The CNN-LSTM accuracy value is 0.08

2. The highest recall value for CNN model is 0.08

3. The highest model CNN Precision Value 1.0

\section{REFERENCES}

[1] E. dwi nurindah Sari, "Analisis Sentimen Nasabah Pada Layanan Perbankan Menggunakan Metode Regresi Logistik Biner, Naïve Bayes Classifier ( NBC ), dan Support Vector Machine ( SVM )," J. Sains Dan Seni Its, vol. 8, no. 2, p. 177, 2019.

[2] T. Rosandy, "PERBANDINGAN METODE NAIVE BAYES CLASSIFIER DENGAN METODE DECISION TREE (C4.5) UNTUK MENGANALISA KELANCARAN PEMBIAYAAN (Study Kasus : KSPPS / BMT AL-FADHILA,” J. Teknol. Inf. Magister Darmajaya, vol. 2, no. 01, pp. 52-62, 2016.

[3] I. C. R. Drajana, "Metode Support Vector Machine Dan Forward Selection Prediksi Pembayaran Pembelian Bahan Baku Kopra," Ilk. J. Ilm., vol. 9, no. 2, pp. 116-123, 2017, doi: 10.33096/ilkom.v9i2.134.116-123.

[4] R. A. Solovyev et al., "Deep Learning Approaches for Understanding Simple Speech Commands," 2020 IEEE 40th Int. Conf. Electron. Nanotechnology, ELNANO 2020 - Proc., pp. 688-693, 2020, doi: 10.1109/ELNANO50318.2020.9088863.

[5] A. S. M. Alharbi and E. de Doncker, "Twitter sentiment analysis with a deep neural network: An enhanced approach using user behavioral information,” Cogn. Syst. Res., vol. 54, pp. 50-61, 2019, doi: 10.1016/j.cogsys.2018.10.001.

[6] Sartini, Analisis Sentimen Twitter Bahasa Indonesia Menggunakan Algoritma Convolutional Neural Network. 2020.

[7] B. Liu, "Sentiment analysis and subjectivity," Handb. Nat. Lang. Process. Second Ed., no. January 2010, pp. 627-666, 2010. 